MATEC Web of Conferences 45, 04013 (2016)

DOI: $10.1051 /$ matecconf/20164504013

C Owned by the authors, published by EDP Sciences, 2016

\title{
Kinematic synthesis of a new 3D printing solution
}

\author{
Hermes Giberti ${ }^{1}$, Enrico Fiore ${ }^{1}$ and Luca Sbaglia ${ }^{2}$ \\ ${ }^{1}$ Department of Mechanical Engineering, Politecnico di Milano, Campus Bovisa Sud - via La Masa 1, 20156 Milano, Italy \\ ${ }^{2}$ Università degli studi di Parma, Dipartimento di ingegneria industriale Parco Area delle Scienze, 181/A, 43124 Parma, Italy
}

\begin{abstract}
Low-cost production of metal parts is a challenge nowadays in the Additive Manufacturing world and new methods are being developed. The MIM technique is an innovative approach for 3D printing. This method requires a machine with suitable kinematics capable of generating the adequate movements.

The object of this article is the kinematic synthesis of a 5Dofs robot, based on two PKM machines, for additive manufacturing in order to compliant with the requirements of this new technology. Robot kinematics have been optimized by genetic algorithm in order to cover the required workspace and the design of the robot and outline of the control system are also given.
\end{abstract}

\section{Introduction}

Additive manufacturing, AM, is constantly growing due to its innovative design capacities. The forecast for this technology indicates that the market in 2017 will be about 5 billion dollars [1]. AM, also known as 3D printing, is based on several technologies based on the same idea: material deposition layer upon layer which task can be done in several ways and with different materials. Table 1 indicates some of the principal companies and technologies used in 3D printing[2],[3].

Tab. 1 Technologies for 3D printing

\begin{tabular}{|l|l|}
\hline Company(foundation year) & 3D printing technology \\
\hline 3D Systems(1986), USA & SLA,SLS \\
\hline Statasys(1992), USA & FDM, Polyjet, WDNM \\
\hline ExOne(2005), USA & Binder jetting \\
\hline Arcam(1997), Sweden & EBM \\
\hline Optomec(1998), USA & Aereoslojet, LENS \\
\hline
\end{tabular}

Among these technologies those related to the metal part production are based on the use of laser or electron beams [4]. Which are very costly. Stratasys is trying to provide an alternative means of production based on FDM [5] but at present there is still no commercial printer available. In order to reduce production costs of metal pieces, MIM technology (metal injection molding) could be a solution. In this way a feedstock made up of metal powder and a polymeric binder would be extruded on a platform layer by layer until completion of the object.

Thereafter the object will undergo heat treatment for the removal of the polymeric binder. Direct extrusion of the material greatly reduces the costs of process. This idea is rather simple but the main problem is related to the quality of the final product. Accordingly the accurate positioning of the worktable is of fundamental importance and this requires particular attention to the design of the mechanical structure.

Most industrial 3D printers have 3Dofs: one for the vertical translation of the platform and two for the movement in a plane of the extrusion head. According to 3D printing technology there can be some slight differences, but usually there are only 3Dofs (Optomec produces a $3 \mathrm{D}$ printer with $5 \mathrm{Dofs}$ ). In general these machines are made up of serial robots based on a Cartesian layout where linear guides, driven by electrical motors, move the worktable and the head.

This article focuses on the kinematic optimization of a 5Dofs robot for a 3D printer based on MIM technology. In our solution the worktable has all the 5 dof while the extrusion head is fixed. This is why the extrusion system is powerful and heavy (about $25[\mathrm{~kg}]$ ), made up of a feeder, a plasticizer and an injector connected to a final nozzle. It would be very difficult to move it quickly and accurately so it was necessary to develop a machine with a worktable completely moveable. This solution with the 5Dofs, 3 translations and 2 rotations, allows the possibility of new paths generation. This could help to solve typical problem of 3D printing like staircase effect, angle deposition [6] or reduce the use of support material [7]. For common 3D printers with only 3Dofs the only way to limit these problems is to reduce the thickness of each layer but this increases the production time and does not resolve the problem related to the use of the support material.

\section{Robot kinematic}

The robot requirements are the starting point of the kinematic synthesis, in particular: cube shape workspace 
of side $100[\mathrm{~mm}]$, positioning precision under $0.1[\mathrm{~mm}]$ and robot 5 Dofs, translations $x-y-z$, rotations roll and pitch until $45^{\circ}$

In order to achieve these requirements, we decided to realize an hybrid structure characterized by two parallel robots in series. The first one carries out the translation $\mathrm{x}-$ $y-z$ movement while he second one the rotation movements. This modular solution allows us to take advantage at a same time of parallel and serial robots characteristics, in particular, a good stiffness and precision from the first one and a rather wide workspace from the second. Note that with a parallel robot it would have been difficult to cover the required workspace mainly due to the large rotation angles required. Another important aspect is that the modular solution allows the use of two robots separately and we can optimize their design and workspace independently. This leads to the solution of two problems with smaller complexity compared to the study of a single 5Dofs robot. The chosen architecture for the first robot is called linear delta. Its purpose is to move the platform over which the second $\mathrm{pkm}$ is mounted. The name of this is agile eye and its purpose is to rotate the worktable where the material is deposited by the extruder. As follows, the kinematics of both the linear delta and agile eye is shown; Studies regarding these two robot architectures are presented in [8],[9]. Inverse and direct kinematic is necessary for the robot kinematic optimization and for the control system development.

\subsection{Linear Delta}

The machine which is responsible of the translational Dofs is a linear delta. This particular architecture is made up of three links of fixed length connecting the mobile platform with three different guides. The actuation is provided by three electrical motors connected to three linear transmission units, guides. Three independent PUS (Prismatic-Universal- Spherical) kinematic chains can be identified. The links are actually three parallelograms; in fact, this particular architecture ensures that the mobile platform is always parallel to the ground. In order to compute the kinematics of this machine two different reference systems are defined, the inertial frame placed on the ground and the Tool-Center-Point (TCP) which is fixed to the mobile platform. Since the links are parallelograms, the axes of the two frames are always parallel whatever the pose of the robot.

\subsubsection{Inverse Kinematics}

With reference to Fig. 1, for the $\mathrm{i}$-th chain it is possible to follow the mathematical steps shown below in order to solve the inverse kinematics:

$$
\begin{aligned}
& \boldsymbol{l}_{\boldsymbol{i}}=\boldsymbol{d}_{\boldsymbol{i}}-q_{i} \widehat{\boldsymbol{u}}_{\boldsymbol{i}} \quad \text { where } \quad \boldsymbol{d}_{\boldsymbol{i}}=\boldsymbol{p}+\boldsymbol{b}_{\boldsymbol{i}}-\boldsymbol{s}_{\boldsymbol{i}} \\
& l_{i}^{2}=\boldsymbol{l}_{\boldsymbol{i}}^{T} \boldsymbol{l}_{\boldsymbol{i}}=\left(\boldsymbol{d}_{\boldsymbol{i}}-\boldsymbol{q}_{\boldsymbol{i}} \hat{u}_{i}\right)^{T}\left(\boldsymbol{d}_{\boldsymbol{i}}-q_{i} \widehat{\boldsymbol{u}}_{\boldsymbol{i}}\right)=\boldsymbol{d}_{\boldsymbol{i}} \boldsymbol{d}_{\boldsymbol{i}}^{T}-\mathbf{2} \boldsymbol{d}_{\boldsymbol{i}}^{T} \widehat{\boldsymbol{u}}_{\boldsymbol{i}} q_{i}+q_{i}^{2}
\end{aligned}
$$

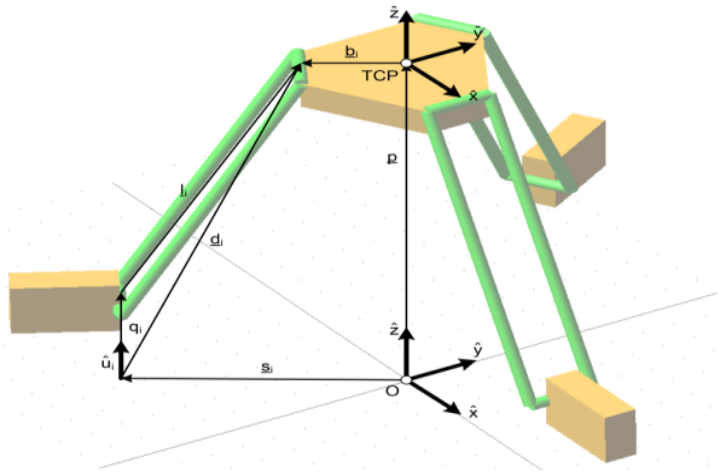

Fig. 1 Linear Delta

$q_{i}=d_{i}^{T} \widehat{u}_{i}-\sqrt{d_{i}^{T}\left(\widehat{u}_{i} \widehat{u}_{i}^{T}-[\mathrm{I}]\right) d_{i}+\boldsymbol{l}_{i}^{2}}$

\subsubsection{Velocity analysis}

Deriving respect to time eq. 2 , it's possible to obtain the expression of the inverse Jacobian matrix:

$$
\begin{aligned}
& 2 \boldsymbol{d}_{\boldsymbol{i}}^{T} \dot{\boldsymbol{d}}_{\boldsymbol{\imath}}-2 \boldsymbol{d}_{\boldsymbol{i}}^{T} \widehat{\boldsymbol{u}}_{\boldsymbol{i}} \dot{q}_{i}-2 q_{i} \widehat{\boldsymbol{u}}_{\boldsymbol{i}}^{T} \dot{\boldsymbol{d}}_{\boldsymbol{\imath}}+2 \dot{q}_{i} q_{i}=0 \\
& \left(d_{i}-\widehat{\boldsymbol{u}}_{i} q_{i}\right)^{T}\left(\dot{\boldsymbol{d}}_{\boldsymbol{\imath}}-\widehat{\boldsymbol{u}}_{\boldsymbol{i}} \dot{q}_{i}\right)=0 \\
& \boldsymbol{l}_{\boldsymbol{i}}^{T}\left(\dot{\boldsymbol{d}}_{\boldsymbol{\imath}}-\widehat{\boldsymbol{u}}_{\boldsymbol{i}} \dot{q}_{i}\right)=0 \\
& \boldsymbol{l}_{\boldsymbol{i}}^{T}\left(\widehat{\boldsymbol{u}}_{\boldsymbol{i}} \dot{q}_{i}-\dot{\boldsymbol{p}}\right)=0 \\
& \widehat{\boldsymbol{n}}_{\boldsymbol{i}}^{T} \widehat{\boldsymbol{u}}_{\boldsymbol{i}} \dot{q}_{i}-\widehat{\boldsymbol{n}}_{\boldsymbol{i}}^{T} \dot{\boldsymbol{p}}=0
\end{aligned}
$$

Considering all the three kinematic chains it is possible to rearrange this expression in a matrix form and obtain the inverse Jacobian:

$$
\begin{aligned}
& \operatorname{diag}\left(\hat{n}_{1}^{T} \hat{u}_{1}, \ldots, \hat{n}_{3}^{T} \hat{u}_{3}\right) \dot{q}-\left[\hat{n}_{1}^{T}, \hat{n}_{2}^{T}, \hat{n}_{3}^{T}\right]^{T} \dot{p}=0 \\
& {[]^{-1}=\operatorname{diag}\left(\hat{n}_{1}^{T} \hat{u}_{1}, \ldots, \hat{n}_{3}^{T} \hat{u}_{3}\right)\left[\hat{n}_{1}^{T}, \hat{n}_{2}^{T}, \hat{n}_{3}^{T}\right]^{T}}
\end{aligned}
$$

\subsection{Agile eye}

The worktable rotational Dofs are obtained through a spherical wrist named agile eye. This PKM is mounted on the mobile platform of the linear delta. In order to solve the kinematics of this manipulator the orientation evaluation of every its circular link is required.

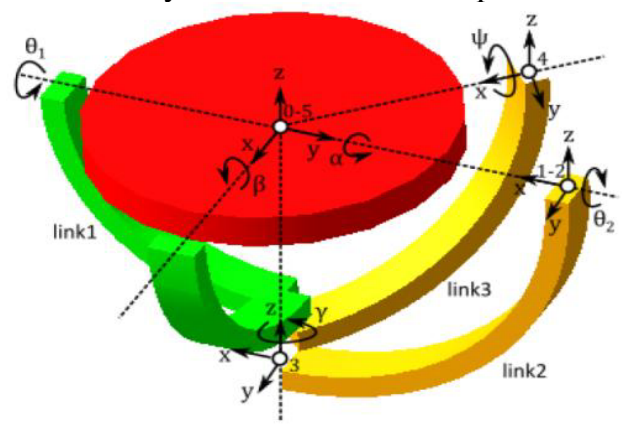

Fig. 2 Agile eye

Figure 2 shows the configuration chosen. The kinematics of this machine depend on the motor position 
and on the position joint of the links and platform. The expression that relates the rotations of the motors $\left(\theta_{1} ; \theta_{2}\right)$ to the roll and pitch ( $\alpha$ and $\beta$ respectively) of the platform is:

$$
\left\{\begin{array}{c}
\theta_{1}=\alpha \\
\theta_{2}=\operatorname{atan}\left(\frac{\sin \alpha-\cos \alpha \sin \beta}{\sin \alpha \sin \beta-\cos \alpha}\right)
\end{array}\right.
$$

\subsubsection{Velocity analysis}

Deriving Eq. 11 with respect to time, you get:

$\left.\dot{\Theta}=\left[\begin{array}{cc}1 & 0 \\ \frac{d \theta_{2}}{d \alpha} & \frac{d \theta_{2}}{d \beta}\end{array}\right]\left\{\begin{array}{l}\dot{\alpha} \\ \dot{\beta}\end{array}\right\}=J_{\theta}\right]^{-1}\left\{\begin{array}{c}\dot{\alpha} \\ \dot{\beta}\end{array}\right\}$

Where $\dot{\Theta}=\left\{\begin{array}{ll}\dot{\theta}_{1} & \dot{\theta}_{2}\end{array}\right\}^{T}$, and $\left[J_{\theta}\right]^{-1}$ represents the inverse Jacobian matrix associated to the rotational motions. The explicit equations have been omitted for the sake of simplicity, but it is rather easy to obtain them deriving the inverse kinematic expressions of $\theta_{1} . \theta_{2}$.

\section{Kinematic optimization}

Due to the modular solution of this project and to the fact that agile eye has no problem in reaching the desired rotation angles, it is possible to optimize only the linear delta kinematics provided that we take into account the behavior of the agile eye. The idea is to increase the working space of the linear delta in order to take into account the position reached by the piece in production caused by the worktable rotations. The result is an equivalent cube into which the center of the movable platform of the linear delta can go.

Linear delta for its own design has no limit for the movement in $\mathrm{z}$ axis direction, so we can study its kinematics in a xy plane. In order to optimize the system first we performed an adimensional study to understand the influence of the geometric parameters of the robot in the definition of its workspace, after a dimensional optimization has been performed to obtain the values of these parameters.

We decided to have the three linear guides of the delta robot at the same distance from the center of the machine. The boundary of the working area is the circle inscribed within the three guides. The robot is obliged not to overpass this circle even if it would be possible to do. Geometric parameters of the optimization are: $D=$ diameter where linear guides are placed, $d=$ mobile platform's diameter, $l=$ legs length and $\alpha=$ angle between guides. The $\alpha$ angle defines the relative position of the guides on circle of $D$ diameter. Considering a guide placed on the $\mathrm{D}$ circle the others two are placed on the same circle but $\pm \alpha$ degrees distant from the previous one. In this way the symmetry of the machine is preserved. To carry on this study a genetic algorithm with a single target has been used. Matlab function $g a$ is used to this purpose.
The matlab function $g a$ works in this way: having chosen a set of $\mathrm{n}$ parameters, 4 in this case, and a specific range for each parameter, the function tries to minimize a desired function value. In our case we wanted to maximize an area so our function was the difference between the target area and the area covered by the robot, minimize this area means maximizing the workspace of the robot [10] [11]. The program works according to the genetic algorithm approach: making a random choice of the set values, inside the specified range, the objective function is evaluated. This process is carried on several times for a number of times, specified by the user, until the first family of the robot (with its own set of parameters) has been created. Later the program proceeds making a second family combining the values of the parameters obtained in the first family. In particular the program gives more importance to the parameters related to the lower values of the objective function found for the first family. The program continues creating several different families of robots and it can stop in different ways, for example because the maximum number of families, defined at the beginning of the optimisation, has been reached or after a situation of stall due to the impossibility to increase the minimization of the objective function.

In this project we had only 4 parameters, which is not a large number; so we made families of 300 members and we set the stop condition when the fiftieth family generation is reached. The objective function is an area evaluation. The robot workspace has been calculated in this way: from the center of the circle (the center of the machine) the platform is moved along a radial path until the position of the platform is reachable in accordance with the inverse kinematics of the machine; thereafter the platform is moved on a circle path for $360^{\circ}$, always checking that the position is reachable according to the kinematic equations. A set of points reached by the platform represents the workspace of the robot, which has to be subtracted from target area. The optimization has to take into account some limitations, in particular: joint limits and force and velocity transmission factors

The joints, used to connect the links ( or robot legs ) from one hand with the movable platform from the other hand and with the slider of the linear guides, have a limited mobility. We have to consider these limits using the genetic algorithms. With respect to the force and velocity transmission factors we set the value three as a limit in order to reduce the force level that the actuation system has to bear. Lower values means that it can bear more weight on the platform, reach higher velocities and using smaller actuators. To evaluate force and velocity transmission factors, an infinite norm of the Jacobian matrix has been used.

$$
\begin{gathered}
\tau_{\text {force }}=\left\|[J]^{T}\right\|_{\infty}<3 \\
\tau_{\text {velocity }}=\left\|[J]^{-1}\right\|_{\infty}<3
\end{gathered}
$$

Notice that the Jacobian, and $\tau_{\text {force }}$ and $\tau_{\text {velocity }}$, depend only on the unitary vectors which defines the space directions of each leg.

\subsection{Adimensional study}

\subsection{Genetic algorithm}


In this first part of the optimization we wanted to understand the influence of the 4 parameters on the robot workspace using an adimensional study. Each parameter was divided by $D$, so the robot diameter was fixed to one and the optimization parameters became three. The legs could change their length from 0.4 to 2 and could vary from 0.2 to 1 . Angle $\alpha$ was free to change between 10 to 170 degrees. Table 3 shows that $d$ has to be as low as possible while $\alpha$ more than $90^{\circ}$, and the length of the legs equal to $D$.

Tab. 2 Results adimensional study

\begin{tabular}{|c|c|}
\hline Parameter & Value \\
\hline$l$ & 1 \\
\hline$d$ & 0.2 \\
\hline$\alpha$ & $95^{\circ}$ \\
\hline
\end{tabular}

\subsection{Dimensional optimization}

Using the results achieved by the adimensional study the kinematics optimization needed to reach the required dimensional workspace has to be performed. The space to cover is a $100[\mathrm{~mm}]$ cube but in order to compensate the movements of the agile eye the dimension of this cube has to be increased. Considering rotations of the worktable up to $45^{\circ}$ and evaluating the displacement of the platform center, it is relatively simple to evaluate that the cube has to be increased from $100 \times 100[\mathrm{~mm}]$ to at least $220 \times 220[\mathrm{~mm}]$. Actually in order to be sure of covering the required workspace at the end of the optimization task, it is a good idea to increase further the estimated value to $300 \times 300$ [mm].

We have to consider that to cover a workspace bigger than what is strictly necessary means to have joints with bigger rotational angle or have a bigger machine with a large dimension $D$. We want to have a compact robot in order to cover the $300 \times 300[\mathrm{~mm}]$ workspace. We set the parameters $d$ to $400[\mathrm{~mm}]$, that is because from the adimensional study we saw how $d$ should be as short as possible and this is the minimum value we need in order to have an adequate space to install the agile eye. In Tab. 3 we see the ranges of the 3 parameters and the results we obtained from the optimization.

Looking to the results we notice how there is no more equality between length legs and diameter $\mathrm{D}$, which is due to the different shape of the workspace we have to cover: a square compared to circle one. As for the angle, we see that it changes between $90^{\circ}$ and $125^{\circ}$. The solutions to the mathematical problem were more than one so the genetic algorithm couldn't converge always to the same solution. Notice that $120^{\circ}$ allows us to have a machine with three symmetry planes, which means a better isotropy inside the workspace.

Moreover we wanted to use the guides not only to move the platform but also as a frame for the robot, as can be seen in figure 4 we decided to fix the angle $\alpha$ equal to $120^{\circ}$ and make the last optimization considering only 2 parameters variable: $l$ and $D$. At the end of the optimization task, the length of the legs is set to $600[\mathrm{~mm}]$ and the diameter D to $913[\mathrm{~mm}]$. In Fig. 3 it is shown the workspace reached with the values found and the actual limits of the revolute joints. We see how we can cover the target square space $220 \times 220[\mathrm{~mm}]$ but not the $300 \times 300[\mathrm{~mm}]$, which is because of the limits in the revolute joints. Anyway the use of a bigger workspace has led to reach a margin on the $220 \times 220[\mathrm{~mm}]$ as we expected.

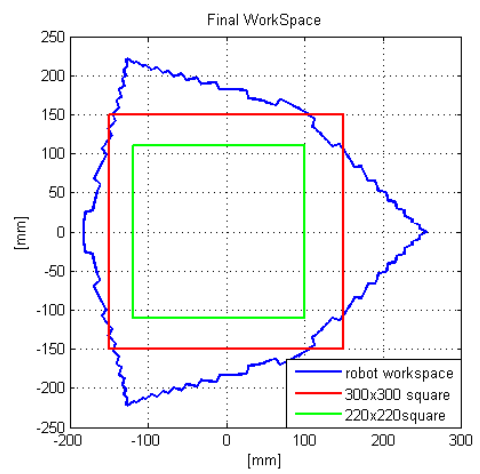

Fig. 3 Linear Delta workspace

Tab. 3 Results dimensional study

\begin{tabular}{|c|c|c|c|c|}
\hline Parameters & range & Results 1 & Results 2 & Results 3 \\
\hline$l[\mathrm{~mm}]$ & $400-1000$ & 660 & 610 & 580 \\
\hline$D[\mathrm{~mm}]$ & $600-1000$ & 0.95 & 0.92 & 0.89 \\
\hline$\alpha$ & $90^{\circ}-170^{\circ}$ & $93^{\circ}$ & $124^{\circ}$ & $117^{\circ}$ \\
\hline
\end{tabular}

\section{Design and control system}

The results obtained in the optimization were used to make the design of the machine. The robot is structured by a mobile platform connected to three parallelograms which are the three legs of the robot and which are connected to the three sliders of the linear guides. The constraints between legs and platform and legs and sliders are rotational joints which allows 2 rotational Dofs. In Fig.4 is shown the complete robot. It's possible to see the three guides where at the bottom there are the motors with their respective gear motors. At the center of the machine there is the mobile platform where the agile eye is mounted. We can see how there are two plates, one at the bottom of the machine and the other on the upper part. These two plates are needed two guarantee the correct mounting of the guides, furthermore the upper plate is necessary to bear the extruder that is fixed on the top of the machine. To increase the all stiffness of the guides and limit their vibration tie beams between each guide will be built.

The all robots will be controlled by means of an industrial control system. This system is principally based on: PLC, Motion control unit until 16 axis and Servo drives fully closed loop integrated. With this kind of system it is possible to control and coordinate all the 7 machine axis: 3 for the Linear Delta, 2 for the Agile Eye and 2 for the extrusion system. The control loops of the Linear Delta and Agile eye can be based either on the encoders of the respective motors or on external sensors.

For instance it is possible to close the position loop for the Linear Delta on the position of the three sliders using magnetic bands along the guides. Direct measure 
of the sliders position allows one to avoid the imprecision due to the backslash between motors and gear motors, and we don't have to consider the elasticity of the belts on which the linear guides are based.

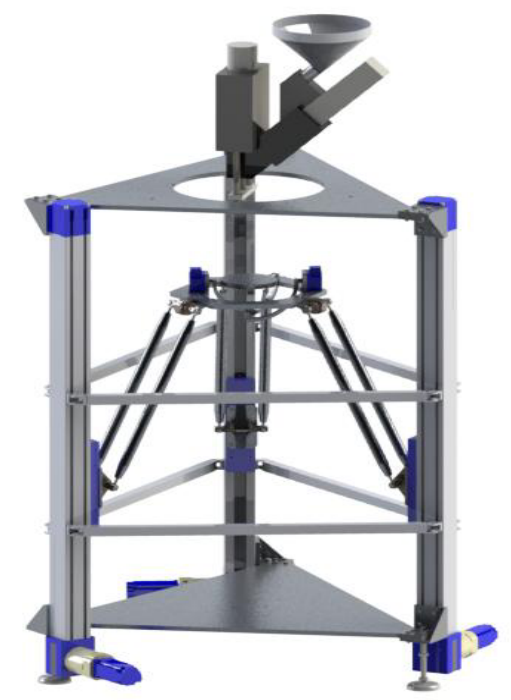

Fig. 4 Machine Design

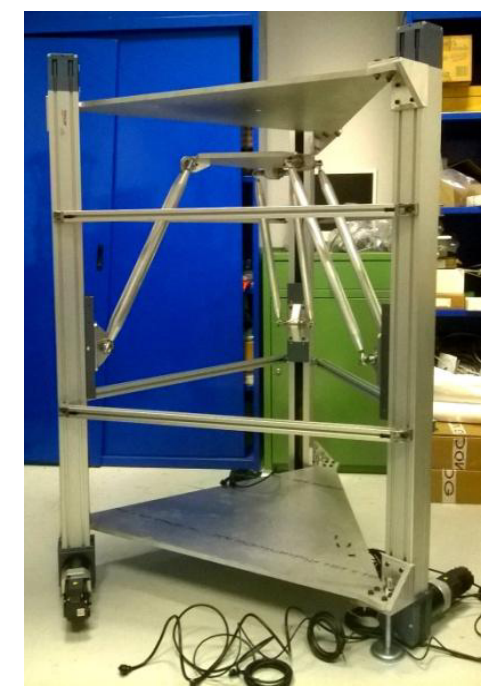

Fig. 5 Machine Protipe

\section{Conclusion}

In this paper we presented a kinematic synthesis of a 5 Dofs robot based on a modular solution given by a linear delta and an agile eye.

According to the results obtained we can see how this new machine concept can give possibilities of movement which are not feasible with classical 3D printer and so it will be possible to develop an innovative system of production based on MIM technology. The robot kinematic allows the generation of paths for the platform which can solve the problems of staircase effect, angle deposition and use of support material. The design of the robot has been made with the aim of covering a specific workspace. It is rather easy following the same procedure shown to cover any desired workspace and so to extend this study to many other situations and different necessities. Simply increasing mobility of revolute joints, which is mainly a question of materials and costs, it will be possible to compensate even bigger rotations of the agile eye, which are already possible, and so develop new paths generation for $3 \mathrm{D}$ printing. The simple design and the commercial control system can simplify the use of this technology and so permit a cheaper production of complex shape metal parts with additive manufacturing which is a growing demand nowadays.

\section{References}

1. B. N. Turner, R. Strong and S. A. Gold, A review of melt extrusion additive manufacturing processes: I. Process design and modeling, Rapid Prototyping Journal, Vol. 20 Iss 3 pp. 192 - 204, (2014)

2. Douglas R. Gress, Ronald V. Kalafsky, Geographies of production in 3D: Theoretical and research implications stemming from additive manufacturing, Geoforum,Vol.60, March 2015, Pages 43-52, (2014)

3. K.P. Karunakaran Alain Bernard S. Suryakumar Lucas Dembinski Georges Taillandier, Rapid manufacturing of metallic objects, Rapid Prototyping Journal, Vol. 18 Iss 4 pp. 264 - 280, (2012)

4. N. Guo, M. C. Leu, Additive manufacturing: technology, applications and research needs, Front Mech Eng, 2013, 8(3): 215-243, (2013)

5. Patent publication number US2009/0314391 A1,assignee Stratasys Inc., system and method for building three-dimensional object with metal based Alloys, (2009)

6. A. Boschetto,L. Bottini, Accuracy prediction in fused deposition modeling, The international journal of advanced manufacturing technology, Volume 73, Issue 5-8, pp 913-928, (2014)

7. W.C. Lee, C.c. Wei, S.C. Chung, Development of a hybrid rapid prototyping system using low-cost fused deposition modeling and five-axis machining, Journal of Materials Processing Technology 214 (2014)

8. M. J. Uddin, S. Refaat, S. Nahavandi, H. Trinh, kinematic and dynamic modeling of a robotic head with linear motors, International Conference on Manufacturing and Management (2004), Queensland, pp 879-884

9. G. Palmieri,M. Callegari, L. Carbonari, M.C. Palpacelli, Design and testing of a spherical parallel mini manipulator, 10th IEEE/ASME International Conference on Mechatronic and Embedded Systems and Applications. IEEE MESA, (2014), pp 1-6

10. D'Antona, G., Davoudi, M., Ferrero, R., Giberti, H. A model predictive protection system for actuators placed in hostile environments, 2010 IEEE International Instrumentation and Measurement Technology Conference, I2MTC 2010 - Proceedings, art. no. 5488111, pp. 1602-1606.

11. Giberti, H., Ferrari, D. A novel hardware-in-the-loop device for floating offshore wind turbines and sailing boats (2015) Mechanism and Machine Theory, 85, pp. 82-105. 